\title{
The neuronal PDE2A3 splice variant is targeted to membranes via dual acylation
} Corina Russwurm¹, Georg Zoidl2, Doris Koesling ${ }^{1}$ and Michael Russwurm*1

\author{
Address: ${ }^{1}$ Institut für Pharmakologie und Toxikologie, Medizinische Fakultät, Ruhr-Universität Bochum, 44780 Bochum, Germany and ${ }^{2}$ Institut \\ für Anatomie, Abteilung für Neuroanatomie und molekulare Hirnforschung, Medizinische Fakultät, Ruhr-Universität Bochum, 44780 Bochum, \\ Germany \\ Email: Michael Russwurm* - michael.russwurm@ruhr-uni-bochum.de \\ * Corresponding author
}

from 4th International Conference of cGMP Generators, Effectors and Therapeutic Implications

Regensburg, Germany. 19-21 June 2009

Published: II August 2009

BMC Pharmacology 2009, 9(Suppl I):P6I doi:I0.I|86/I47|-22I0-9-SI-P6I

This abstract is available from: http://www.biomedcentral.com/I47I-22 I0/9/SI/P6 I

(c) 2009 Russwurm et al; licensee BioMed Central Ltd.

\section{Background}

The cyclic nucleotide second messengers cAMP and cGMP play key roles in mammalian signal transduction. Their intracellular levels are tightly controlled by the rate of synthesis and degradation. Hydrolysis of the cyclic nucleotides is dependent on the activity of phosphodiesterases (PDE) of which to date 11 families have been identified. The cGMP-stimulated cGMP/cAMP phosphodiesterase 2 is encoded by one gene, which has been shown to be spliced into three variants that have been cloned from different species. Besides the ubiquitous soluble PDE2A1, two membrane associated forms, PDE2A2 and PDE2A3, have been described. The PDE2A splice variants only differ in short stretches of amino acids at their very $\mathrm{N}$ terminus. This region has previously been suggested to determine the subcellular localization of the enzyme, but data about the mechanism underlying membrane attachment of PDE2A3 are lacking.

\section{Results}

Here, we show that concurrent myristoylation and palmitoylation targets PDE2A3 to synaptic membranes. In metabolic labeling studies, PDE2A3 incorporated ${ }^{3}[\mathrm{H}]$ myristate, whereas mutation of the myristoyl recipient $\mathrm{Gly}^{2}$ prevented radiolabeling. Additionally, and in contrast to the wild type enzyme, the non-myristoylated mutant was completely soluble when expressed in HEK293 or PC12 cells. As myristoylation alone is rarely sufficient for stable membrane attachment of proteins, the cysteines 5 and 11 as possible candidates for palmitoylation were also mutated. This partially solubilized the enzyme and led to an accumulation in the ER/Golgi compartment, as shown by fluorescence microscopy. By generating isoform-specific antibodies, we were able to confirm on the protein level the widespread expression of the soluble PDE2A1 in mouse tissues. More importantly, we demonstrate for the first time that PDE2A3 is predominantly expressed of in mouse brain where it is attached to synaptosomal membranes. In primary cultures of hippocampal neurons an expression pattern overlapping with synaptophysin was observed, suggesting a presynaptic localization.

\section{Conclusion}

Our results suggest that the neuronal PDE2A3 is dually acylated and thereby targeted to synaptic ends, where PDE2A3 can mediate interplay between CAMP and CGMP dependent signaling pathways. 